\title{
PIN diode driver for NMR and MRI
}

Johansen, Daniel Højrup; Albannay, Mohammed M.; Petersen, J. R.; Zhurbenko, V.; Ardenkjær-Larsen, J. H.

\section{Published in:}

Journal of Magnetic Resonance

Link to article, DOI:

10.1016/j.jmr.2019.01.017

Publication date:

2019

Document Version

Peer reviewed version

Link back to DTU Orbit

Citation (APA):

Johansen, D. H., Albannay, M. M., Petersen, J. R., Zhurbenko, V., \& Ardenkjær-Larsen, J. H. (2019). PIN diode driver for NMR and MRI. Journal of Magnetic Resonance, 300, 114-119.

https://doi.org/10.1016/j.jmr.2019.01.017

\section{General rights}

Copyright and moral rights for the publications made accessible in the public portal are retained by the authors and/or other copyright owners and it is a condition of accessing publications that users recognise and abide by the legal requirements associated with these rights.

- Users may download and print one copy of any publication from the public portal for the purpose of private study or research.

- You may not further distribute the material or use it for any profit-making activity or commercial gain

- You may freely distribute the URL identifying the publication in the public portal

If you believe that this document breaches copyright please contact us providing details, and we will remove access to the work immediately and investigate your claim. 


\title{
PIN Diode Driver for NMR and MRI
}

\author{
D.H. Johansen ${ }^{\mathrm{a}, *}$, M.M. Albannay ${ }^{\mathrm{a}}$, J.R. Petersen ${ }^{\mathrm{a}}$, V. Zhurbenko ${ }^{\mathrm{a}}$, J.H. Ardenkjær-Larsen ${ }^{\mathrm{a}, \mathrm{b}}$ \\ ${ }^{a}$ Technical University of Denmark, Department of Electrical Engineering, Ørsteds Plads 349, Kgs. Lyngby, Denmark \\ ${ }^{b}$ General Electric, Brøndby, Denmark
}

\begin{abstract}
Designing custom coils for magnetic resonance systems, such as nuclear magnetic resonance (NMR) spectrometers and magnetic resonance imaging (MRI) scanners, often entails using non-standard configurations of the transmit-receive (T/R) switch and Q-spoiling circuits. The built-in drivers of commercial NMR and MRI systems are, typically, only reconfigurable within a narrow application range (if at all). Thus, the built-in driver may not be able to properly control the custom T/R switches and Q-spoiling circuits when using custom built coils. We present a PIN diode driver which functions in both an MRI scanner and NMR spectrometer. The PIN diode driver is based on readily available discrete components and achieves switching times for the reverse and forward bias states (transmit on and off) of $2 \mu$ s and $0.4 \mu$ s respectively. Hence, this work enables a higher degree of customization of the RF switching circuits in an MR system and is potentially of interest for designers of custom coils for both NMR spectrometers and MRI scanners.
\end{abstract}

Keywords: Active decoupling; driver; MRI; NMR; switch; PIN diode; TR switching; Q-spoiling.

\footnotetext{
Declarations of interest: None.

* Corresponding author

Email addresses: dhjo@elektro.dtu.dk (D.H. Johansen), mohalb@elektro.dtu.dk (M.M. Albannay), janr@elektro.dtu.dk (J.R. Petersen), vz@elektro.dtu.dk (V. Zhurbenko), jhar@elektro.dtu.dk (J.H. Ardenkjær-Larsen)
} 


\section{Introduction}

In a magnetic resonance (MR) system it is vital to have reliable switching between transmit and receive states of the radio frequency (RF) front-end. The majority of such circuits for switching in an MR system are based on positive-intrinsic-negative (PIN) diodes [1-4]. Micro-electromechanical systems (MEMS) [5] and transistor [6] based switches are, however, slowly emerging as an alternative. The two most commonly used applications for PIN diodes are transmit-receive $(\mathrm{T} / \mathrm{R})$ switches and Q-spoiling circuits (also referred to as active decoupling or active detuning circuits). T/R switches and Q-spoiling circuits have been discussed in depth in [1,7]. However, the treatment of PIN diode driver circuits (just driver moving forward) for MR systems have largely escaped scrutiny, despite the fact that drivers are present in all nuclear magnetic resonance (NMR) spectrometers and magnetic resonance imaging (MRI) scanners. Even though drivers are readily available in different commercial systems, when designing custom coils, a higher degree of flexibility in controlling, for example, the T/R switch and Q-spoiling circuits is often imperative for optimal performance. This work discusses the requirements for a driver in an MR system and presents a design capable of $\mu \mathrm{s}$ switching for both forward and reverse bias.

A PIN diode is made by sandwiching an insulating material between a P-and N-doped semiconductor. At RF and microwave frequencies a PIN diode acts as a non-linear current-controlled resistor [8]. When forward biased a PIN diode achieves resistances in the order of 0.1-0.5 $\Omega$ (depending on the forward current) with a parasitic parallel capacitance of approximately $0.1-2 \mathrm{pF}$ (dictated by the size of the PIN diode). In reverse bias, the resistance of the PIN diode increases to several $\mathrm{k} \Omega$. The exact reverse bias resistance is highly dependent on the reverse voltage. At $0 \mathrm{~V}$ reverse bias the resistance is usually in the range of $5-10 \mathrm{k} \Omega[9]$. The primary problem when having to switch PIN diodes is an inherent non-linear charge storage characteristic resulting in having to source or sink a large current when transitioning from forward to reverse bias or vice-versa.

Drivers are available commercially for cellular applications where the current sourcing capability is limited at $100 \mathrm{~mA}$ with a switching speed of up to $1650 \mathrm{~ns}[10]$. [11, 12] describe an implementation, which is capable of delivering peak currents in excess of $1 \mathrm{~A}$ at switching times of $15 \mathrm{~ns}$. In [13], a driver based on operational amplifier (opamp) technology is presented showing switching times in the order of 200 ns. Forlani and Fresia [14] describe a driver capable of delivering $10 \mathrm{~mA}$ to a PIN diode with a switching time of $1.6 \mathrm{~ns}$. Harvey [15] presents a design capable of delivering $120 \mathrm{~mA}$ at a switching time of $6 \mathrm{~ns}$. van Niekerk and van der Walt [16] show a design with $200 \mathrm{~mA}$ current sourcing. The switching time is up to $7 \mu \mathrm{s}$. In [17] the presented driver switches in $3 \mu \mathrm{s}$ with a current of $30 \mathrm{~mA}$. For MRI, a design is presented by [18]. The design is based on [19] and is capable of switching in approximately 4 us. Brunner et al. [20] presents a complete differential $\mathrm{T} / \mathrm{R}$ switch, including a differential driver for NMR and MRI. The driver is based on the opamp design in [13] and is capable of sourcing up to $1.8 \mathrm{~A}$ with a switch time of approximately $700 \mathrm{~ns}$.

Two key issues with the currently available drivers are: 1) The drivers require a specific series resistor for a given output voltage (which determines the current through the PIN diode). Since the resistor determines the output voltage, the sourcing current is vulnerable to changes in the knee voltage of the PIN diode. For instance, given a certain Q-spoiling switch employing multiple PIN diodes in series, the resistance regulating the biasing current needs to be recalculated and changed in the driver. 2) Low and/or unclear current sourcing and sinking capabilities.

In this work, the two problems described above are addressed by designing a driver, which features a reconfigurable regulated forward bias current between $65 \mathrm{~mA}$ and $1.4 \mathrm{~A}$, with a reverse bias switching time of $0.4 \mu \mathrm{s}$.

\section{Methods}

For the driver to work in both an NMR spectrometer and MRI scanner it is required that the driver can trigger on both a voltage-to-voltage and current-to-voltage input (transmit on-to-off). The voltage-voltage input is typically supplied from a microprocessor (e.g. $5 \mathrm{~V}$ and $0 \mathrm{~V}$ for the transmit on and off state respectively) and the current-voltage input is the Q-spoiling signal from the MRI scanner (e.g. $100 \mathrm{~mA}$ current forward and $-5 \mathrm{~V}$ reverse bias). When the input to the driver transitions from low to high, the 


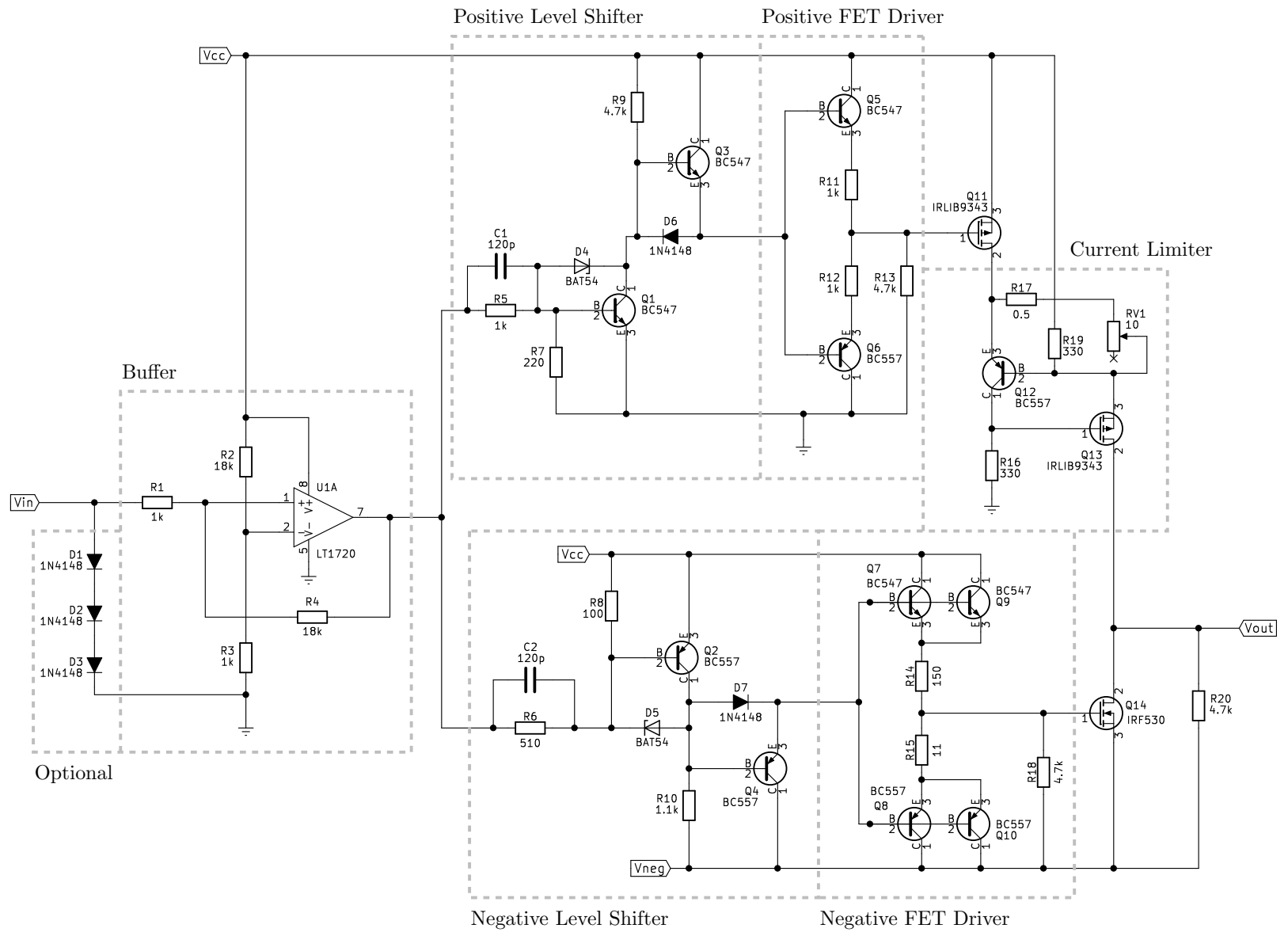

Figure 1: Schematic of the PIN diode driver.

\subsubsection{Buffer}

The buffer consists of a Schmitt trigger ensuring that the PIN driver does not flicker if noise is present on Vin. It is a standard non-inverting topology using the LT1720 comparator (U1A), which is a rail-to-rail

output of the driver needs to transition from a reverse bias of $-5 \mathrm{~V}$ to a forward bias between 100-500 mA and vice versa. Even though the driver is applicable to both NMR and MRI systems, it is primarily designed for an MRI system where a single positive supply of $10 \mathrm{~V}$ is available. We target switching times from forward to reverse bias in under $1 \mu \mathrm{s}$ and from reverse to forward bias below $10 \mu \mathrm{s}$.

\subsection{Driver Circuit}

The schematic of the driver is shown in Fig. 1. The first part of the driver is a buffer ensuring better input jitter stability. The optional diodes are required when triggering the forward bias state of the driver using a current rather than a voltage (required when the Q-spoiling signal from the scanner has to trigger the driver). The buffer triggers when Vin transitions between approximately $0.25 \mathrm{~V}$ and $0.5 \mathrm{~V}$. The positive level shifter inverts the output of the buffer and controls the positive field effect transistor (FET) driver which controls Q11. Q11 is an IRLIB9343 P-channel metal oxide semiconductor FET (MOSFET) and is used as a high side switch. The negative level shifter likewise inverts the signal from the buffer and controls the negative FET driver, which toggles Q14. Q14 is an IRF530 N-channel MOSFET that is operated as a low side switch. To ensure that the sourcing current of the driver is independent of the load connected to Vout, a discrete current limiter is used. 
and single supply OpAmp. When Vin transitions above an upper threshold voltage $V_{\mathrm{h}}$ the buffer output $V_{\mathrm{oh}}=$ Vcc. Vice versa, when Vin transitions below a lower threshold $V_{1}, V_{\mathrm{ol}}=0 . \mathrm{R} 1, \mathrm{R} 2, \mathrm{R} 3$, and R4 are found by solving [21]

$$
\begin{aligned}
R 1 & =\frac{R_{4}\left(V_{\mathrm{h}}-V_{\mathrm{l}}\right)}{V_{\mathrm{oh}}-V_{\mathrm{ol}}}, \\
V_{\mathrm{ref}} & =\frac{V_{\mathrm{h}} V_{\mathrm{ol}}-V_{1} V_{\mathrm{oh}}}{V_{\mathrm{h}}-V_{\mathrm{l}}-V_{\mathrm{oh}}+V_{\mathrm{ol}}},
\end{aligned}
$$

where $V_{\text {ref }}$ is the voltage at pin 2 of U1A. $V_{\text {ref }}$ is defined by the voltage division between R2 and R3. Hence, the threshold voltage are approximately $V_{\mathrm{h}}=0.5 \mathrm{~V}$ and $V_{\mathrm{l}}=0.25 \mathrm{~V}$.

\subsubsection{Level Shifters}

The level shifters convert the output of the buffer (pin 7 of U1A) from $\{0, \mathrm{Vcc}\} \mathrm{V}$ to $\{\mathrm{Vcc}, 0\} \mathrm{V}$ or $\{$ Vcc, Vneg\} V for the positive and negative level shifter respectively. Hence, the level shifters also work as inverters.

When the buffer output voltage transitions low, current runs through the base of Q2 and R6. In turn, the voltage at the base of Q4 rises, forward biasing D5, and subsequently dividing the current through R6 between D5, R8, and the base of Q2. Because D5 is a Schottky diode, having a lower knee voltage $(0.4 \mathrm{~V})$ than the transistor $(0.7 \mathrm{~V})$, it defines the operating point of Q2 and thus ensures that Q2 is not in saturation. Hence, D5 ensures faster switching because Q2 is not in saturation. Further, D5 also mitigates the variance of the conduction voltage of Q2 and thus ensures a uniform performance of the level shifter across multiple devices. D7 protects Q4 by ensuring that the base emitter voltage does not exceed the reverse knee voltage of Q4 $(5 \mathrm{~V})$. When the output of the buffer goes high, C2 discharges into the base of Q2 and D5, Q2 is closed (swiftly due to C2), R10 pulls the base of Q4 to Vneg, and Q4 thus opens and pulls down the output of the negative level shifter to Vneg minus the base emitter voltage of Q4 $(0.7 \mathrm{~V})$.

The positive level shifter works the same as the negative level shifter but is 'flipped'. When the output of the buffer is high, $\mathrm{C} 1$ discharges primarily into the base of Q1 turning it on quickly. Current thus flows through R5 and the base of Q1, opening Q1, pulling down the base of Q3, and pulling down the output of the positive level shifter to ground. When the buffer output drops to zero Q1 is turned off, R9 pulls the base of Q3 to Vcc, Q3 is then turned on and the output of the positive level shifter rises to Vcc minus the base emitter voltage of Q3.

\subsubsection{FET Drivers}

The FET drivers are standard BJT push-pull emitter followers based on the N-type BC547 and P-type BC557 transistors. In the positive FET driver, R11 limits the in-rush current to the gate of Q11 and thus controls the switch off time of Q11. R12 limits the out-rush current from the gate of Q11 determining the turn on time of Q11. In the negative FET driver, R15 determines the out-rush current from the gate of Q14 and hence the turn off time of Q14. R14 limits the in-rush current to the gate of Q14 and hence the turn on time of Q14. Two parallel transistors is utilized on the high (Q7 and Q9) and low (Q8 and Q10) side of the negative FET driver because the peak currents when switching a physically large FET driven hard into saturation can exceed the maximum safe operating current of the BJ547 and BJ557 transistors.

\subsubsection{Current Limiter}

If R19 is excluded, the current limiter is fairly well-known and not very fast. As the voltage on the drain of Q11 rises the source voltage of Q13 also rises and thus turns on Q13, because the gate of Q13 is grounded via R16. As Q13 turns on and draws current through R17 and RV1 the emitter base voltage of Q12 approaches the conduction voltage of Q12. As Q12 starts to conduct, current in R16 increases, increasing the gate voltage of Q13 and again decreasing the current in Q13. Hence, the regulated current output from the source of Q13 is defined by Ohm's law as 


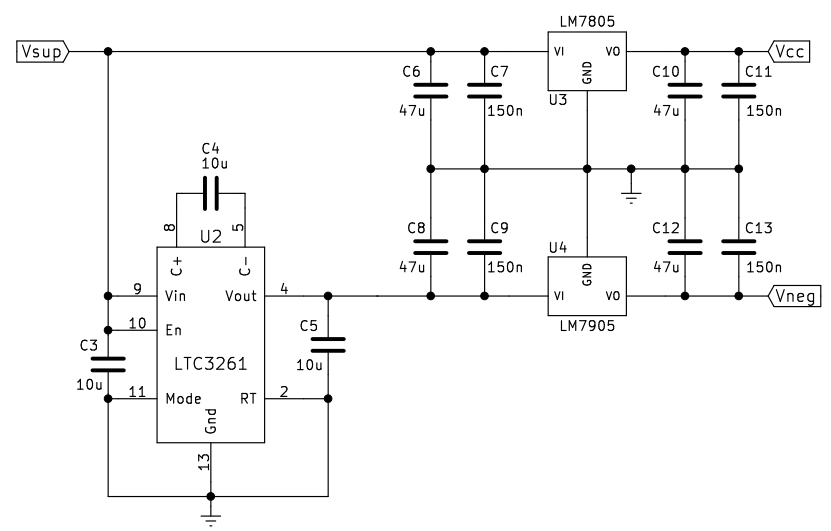

Figure 2: Schematic of the power supply for the PIN diode driver.

$$
I_{\mathrm{out}}=\frac{V_{\mathrm{eb}}^{(\mathrm{Q} 12)}}{\mathrm{R} 17+\mathrm{RV} 1} .
$$

The BC557 has an approximate conduction voltage of $0.7 \mathrm{~V}$ and thus the minimum and maximum current is $67 \mathrm{~mA}$ and $1.4 \mathrm{~A}$ respectively. R19 is added to prevent Q13 from going out of conduction (by adding a small quiescent current of approximately $10 \mathrm{~mA}$ ) and thus decrease the turn on time of the current limiter.

\subsection{Power Supply}

The driver circuit requires three voltages: Vcc, Vneg, and ground. The schematic in Fig. 2 shows the power supply used for the driver. To generate Vcc, Vsup is regulated to $5 \mathrm{~V}$ using an LM7805 linear regulator (U3). The minimum and maximum input voltage to $\mathrm{U} 3$ is $7 \mathrm{~V}$ and $25 \mathrm{~V}$ respectively. Because the driver is designed to operate on a single supply from an MRI scanner an LTC3261 charge pump (U2) is used. U2 converts Vsup to -Vsup on VI of U4 and operates from a Vsup ranging from $4.5 \mathrm{~V}$ to $32 \mathrm{~V}$ with a maximum of $100 \mathrm{~mA}$. The negative voltage from U2 is regulated to $-5 \mathrm{~V}$ using an LM7905 linear regulator (U4) which operates with an input voltage from $-7 \mathrm{~V}$ to $-25 \mathrm{~V}$.

\subsection{Implementation}

The driver and power supply is implemented on a double sided FR-4 substrate with a thickness of $1.5 \mathrm{~mm}$, relative permittivity of approximately 4.2, and copper thickness of $35 \mathrm{um}$. The layout is made in KiCad (files available online) and manufactured using an in-house process. Our in-house process does not feature plated through holes. Vias are made using $0.6 \mathrm{~mm}$ diameter copper rivets. The complete driver is seen in Fig. 3.

\subsection{Bench Characterization}

The bench characterization consists of measuring the on and off switching times of the driver when loaded with a number of PIN diodes at different forward bias currents. This is accomplished using an arbitrary waveform generator to trigger the driver and an oscilloscope featuring at least two channels. One channel of the oscilloscope is connected to Vin along with the output of the arbitrary waveform generator. The second channel of the oscilloscope is connected to Vout. It is vital that the grounding lines of the arbitrary waveform generator and the oscilloscope channels are kept as short as possible to prevent ringing. 


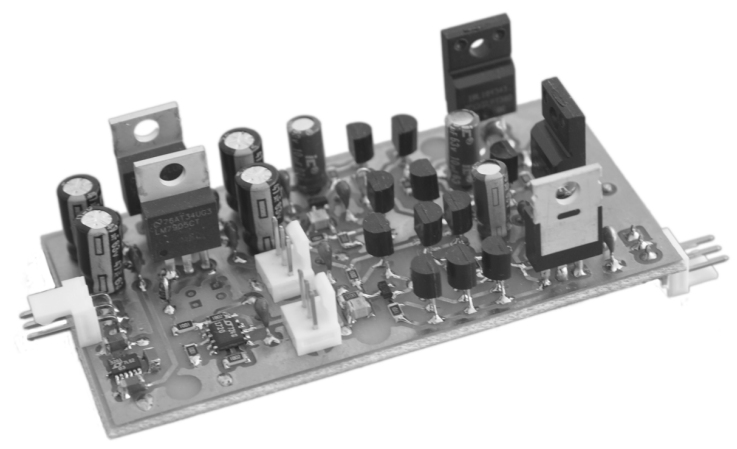

Figure 3: Picture of the PIN diode driver.

\subsection{Imaging and Spectroscopy Experiments}

Imaging of ${ }^{13} \mathrm{C}$ was conducted on a $3 \mathrm{~T}$ clinical scanner (GE Healthcare MR750). A cylindrical phantom filled with ethylene glycol mixed with $17 \mathrm{~g} / \mathrm{L} \mathrm{NaCl}$ was used (to provide loading). The length of the sample is $10 \mathrm{~cm}$ and it has a diameter of $3 \mathrm{~cm}$. A chemical shift imaging (CSI) sequence was used with a repetition time of $250 \mathrm{~ms}$, slice thickness of $4 \mathrm{~cm}$, and field-of-view (FOV) of 14-by-14 $\mathrm{cm}$ in the axial plane. The transmit coil is a clamshell type from RAPID Biomedical. The receive coil is a self-made surface coil which is rectangular with a length and width of 6 and $8 \mathrm{~cm}$ respectively. The edges have been metered at a length of $1 \mathrm{~cm}$. The unloaded and loaded Q-factors are 381 and 172, respectively. Hence, the unloaded to loaded Q-factor is approximately 2.2.

Solid-state dynamic nuclear polarization (DNP) NMR experiments were conducted in a 6.7 T polarizer magnet using a Varian DirectDrive 400 spectrometer. The spectrometer's T/R switch is equipped with three MA4P404-28 PIN diodes biased between $+5 \mathrm{~V}$ and $-15 \mathrm{~V}$ (with the built-in driver) during transmission and reception mode, respectively. A current of $100 \mathrm{~mA}$ biases each diode whilst pulsing thereby minimizing the insertion loss to the sample coil and maximizing receiver preamplifier isolation. Centered in an $\mathrm{RF}$ coil is a $50 \mu \mathrm{L}$ sample of $14 \mathrm{M}\left[1-{ }^{13} \mathrm{C}\right]$ pyruvic acid containing $30 \mathrm{mM}$ of the trityl radical AH111501. The RF coil exhibits an unloaded Q-factor of approximately 45. Due to very low sample loading $(\tan \delta<0.0002)$, the unloaded to loaded Q-factor is approximately 1. The sample was cooled in a helium bath to $1.4 \mathrm{~K}$ and irradiated with $187.96 \mathrm{GHz}$ microwaves. The microwave source is based on an actively multiplied Gunn diode oscillator from Quinstar Technology and a 200X2R4 frequency doubler from VDI. Microwave irradiation is seized after $0.5 \mathrm{~h}$ to acquire a free induction decay (FID). A total of 20 FIDs were acquired using both the Varian and self-built driver. The data is acquired using a spectral bandwidth of $5 \mathrm{MHz}$, a flip angle of 0.36 degrees, and 2048 number of points.

\section{Results}

Fig. 4 shows the measured switching times of the driver on the bench utilizing different forward bias currents and number of PIN diodes in series. Switching the driver to forward bias, when a single PIN diode is connected to the output, when sourcing $100 \mathrm{~mA}, 250 \mathrm{~mA}$, and $500 \mathrm{~mA}$ results in a steady state after approximately $2 \mu \mathrm{s}$ (Fig. 4a). Switching the driver to reverse bias with a single PIN diode connected to the output, for the three sourcing currents mentioned above, results in a steady state after approximately $0.4 \mu \mathrm{s}$ which is achieved at $500 \mathrm{~mA}$ (Fig. 4b). At $100 \mathrm{~mA}$ the reverse bias steady state is reached in approximately $0.28 \mu \mathrm{s}$.

The forward bias switching time when sourcing $100 \mathrm{~mA}$, but adding PIN diodes in series, is seen in Fig. 4c. Up to four series PIN diodes has been added resulting in steady state switch on times of approximately $1.3 \mu \mathrm{s}$, $1.5 \mu \mathrm{s}, 1.7 \mu \mathrm{s}$, and $2.1 \mu \mathrm{s}$ when using 1, 2, 3, and 4 series connected PIN diodes on the output of the driver respectively. As expected, the steady state voltage rises with additive knee voltages of the PIN diodes: $0.7 \mathrm{~V}, 1.4 \mathrm{~V}, 2.1 \mathrm{~V}$, and $2.8 \mathrm{~V}$ for $1,2,3$, and 4 series connected PIN diodes. As the overshoot voltage 


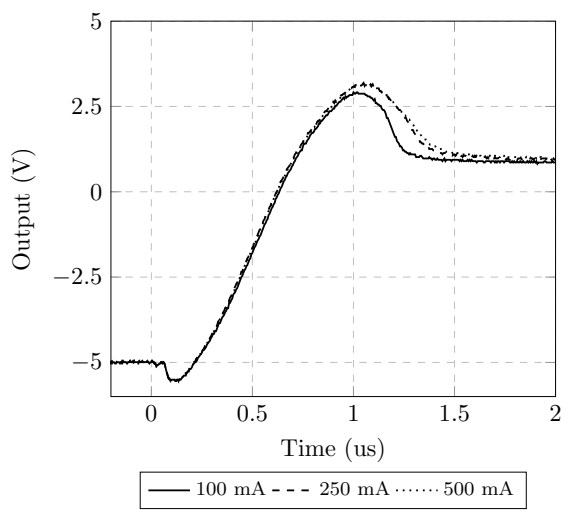

(a)

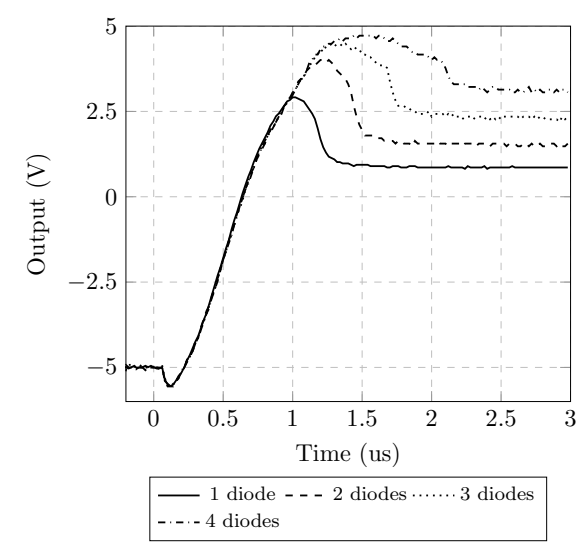

(c)

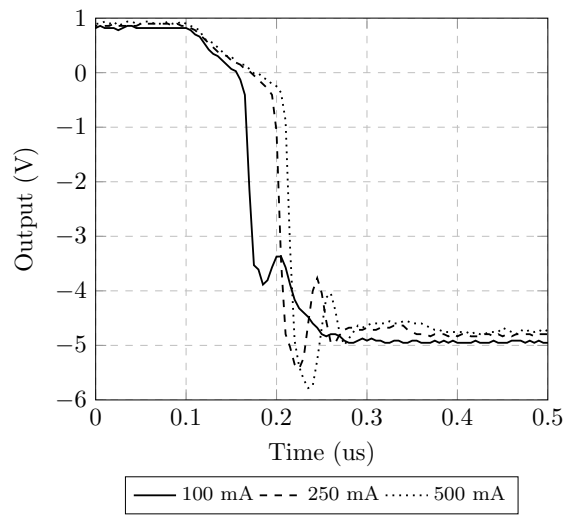

(b)

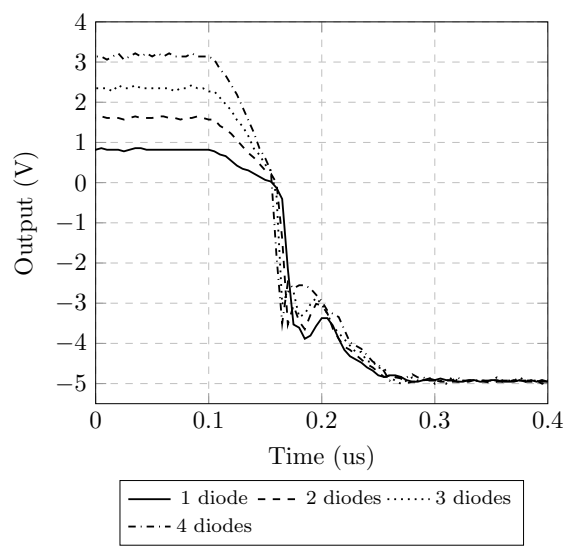

(d)

Figure 4: Bench measurements showing the switch on and off timing of the PIN diode driver. (a) shows the switch on timing. (b) shows the switch off timing.

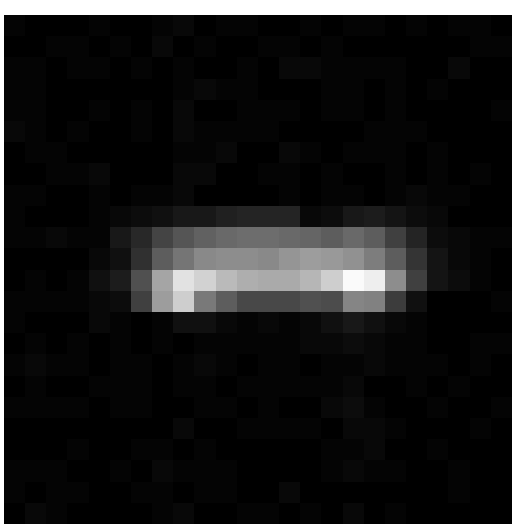

(a)

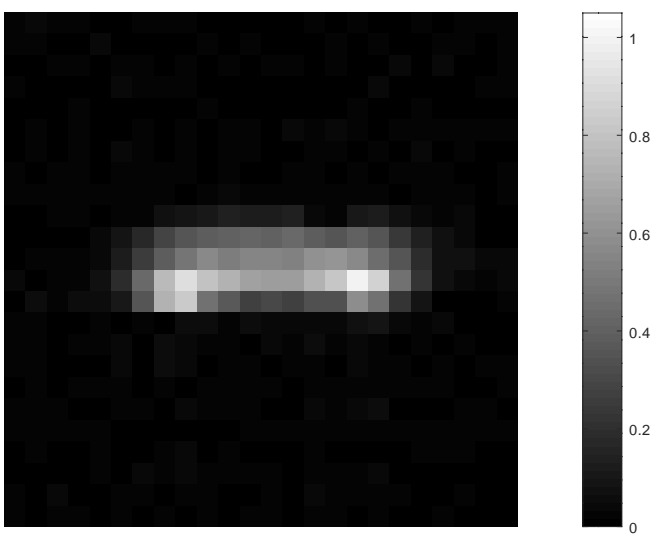

(b)

Figure 5: Scanner measurement showing an image of the cylindrical phantom with a) the scanner's built-in driver and b) the driver presented in this work.

approaches Vcc the linear increase in switching time breaks down and the switching time increases. The reverse bias switching time when using multiple series diodes is seen in Fig. 4 d. It is found that the reverse 


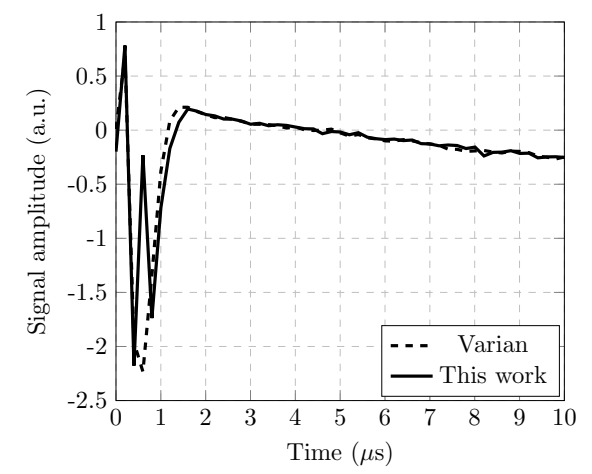

Figure 6: Measured FID (first $10 \mu \mathrm{s}$ ) using the built-in PIN driver of the Varian spectrometer and the one presented in this work.

bias switching time is independent on the number of series connected PIN diodes (at least up to 4) showing an almost constant reverse bias switching time of approximately $0.27 \mu \mathrm{s}$.

Imaging results are seen in Fig. 5. Using the scanner's built-in driver an SNR of approximately 42 was measured. Using the driver presented here an SNR of approximately 43 was measured. Hence, there is no significant difference between the two measurements.

The first $10 \mu$ s of the measured FID using the spectrometer is shown in Fig. 6. No significant difference between the two measurements are observed, exhibiting an approximate switching time of $1.5 \mu$ s. The SNR is 1256 and 1331 using the Varian and self-built driver, respectively. This amounts to an approximately $6 \%$ increase when using the self-built driver.

\section{Discussion}

It was expected that the imaging experiments should show no significant difference in SNR when using the built-in or self-built driver. This observation is only true when using the CSI sequence, or other sequences with similar timing requirements. To extend the usage of the driver to more timing critical applications, the driver was also applied to a solid-state spectroscopy experiment. Here, no significant SNR difference was observed either. Hence, even though the self-built driver does not supply a $-15 \mathrm{~V}$ negative supply compared to the Varian, similar SNR values are achieved. This indicates that the self-built driver is indeed as good as both the built-in driver in the MRI scanner and the NMR spectrometer. The driver presented in this work does not enable e.g. zero echo time imaging, as this would, likely, require a complete redesign of the T/R switch. The primary objective of this work was, however, to enable a higher degree of flexibility in utilizing custom $\mathrm{T} / \mathrm{R}$ switches and Q-spoiling circuits.

The switching time is in this work defined using the MA4P1250NM PIN diode connected directly to the output of the driver. Hence, required circuitry of a $\mathrm{T} / \mathrm{R}$ switch or Q-spoiling circuit such as feeding inductor(s) and decoupling capacitor(s) are not included in the switching time characterized on the bench. We believe that the switching time, when characterized on the bench using a PIN diode while omitting the use of other circuitry, shows the capability of the driver to handle the non-linear current sourcing and sinking of the PIN diode. Even though the switching time is not truly characterized only by the PIN diode it does represent a best case scenario. It is important to recognize that by omitting the use of feed inductors and decoupling capacitors on the bench, also eliminates ringing and the potential use of a snubber circuit. A snubber circuit typically consists of a series resistor, shunt capacitor filter which filters the ringing above certain frequencies. It also slows down the switching time of the driver. The snubber circuit is omitted in this work, but is thoroughly described in [22], and can easily be added to the driver.

The forward bias switching time of the driver can be lowered by further increasing the supply voltage to the positive level shifter, positive FET driver, and Q11. When the driver switches to forward bias, due to the current limiter having a finite regulation time, the output overshoots. This overshoot can be used 
actively in driving the non-linear current sourcing of the PIN diode making it switch faster. The trade-off is a higher steady state power consumption. Q13 will dissipate the majority of the power since this is what regulates the voltage resulting in a given current. Hence, depending on the increase in voltage supply, Q13 may need additional cooling and/or swapped for another transistor entirely.

Decreasing the reverse bias switching time, which is perhaps the most critical, might be achieved by using dedicated logic level transistors which are usually faster than the multi-purpose transistors used in this work. The main issue with logic level transistors is their lower power handling capabilities. Hence, even though the level shifters and FET drivers were updated with logic level transistors, it might not be possible to drive the rather high amount of current when having to switch high power MOSFETs away from deep saturation.

Another strategy for decreasing the reverse bias switching time is to use a lower negative voltage supply for the negative level shifter, negative FET driver, and Q14. This also has the advantage of increasing the resistance of the PIN diode. Now, even though a higher negative voltage may help reduce the reverse bias switching time, it also depends on the slew rate of the driver itself seeing this is finite. Hence, depending on the PIN diodes non-linear current sinking, it could be advantageous to increase the negative supply voltage. However, it should be determined on a case by case basis. The GE scanner, for which this driver was primarily designed, uses a $-5 \mathrm{~V}$ reverse bias and thus is what is used for this driver.

Now, increasing the positive and negative supply voltages might look straight forward. However, it is highly dependent on the maximal drain-source and base-emitter voltages of the transistors. If these maximum ratings are exceeded either other transistors must be utilized or a stacked transistor design is required. The stacked transistor design is not presented in this work since it is well described in [23].

Malfunction detection has not been implemented. By using a differential opamp configuration over the static resistor in the current limiter (R17) to detect the voltage, the current is easily calculated using Ohm's law. The problem with this approach occurs if Q14 or R20 breaks into a short. The current then flows through Q14 and/or R20 rather than into the PIN diode. The safest approach is to add a small series resistor in the output and thus detect the voltage across, again using a differential opamp configuration.

The standard components used to implement the driver are magnetic. This is of course not ideal. Usually, the culprit is the package of which the leads have a layer of nickel deposited. If it is possible to acquire the dies before packaging, custom packing/direct bonding to the printed circuit board (PCB) might solve the problem. However, it would be immensely expensive and the lead time would be very long. As such, we make sure that the driver is placed at least $0.5 \mathrm{~m}$ away from the region of interest in the MRI scanner. No shimming problems were observed.

Finally, the driver presented in this work is single ended. Brunner et al. [20] showed that if sub- $u$ s switching of a high power $\mathrm{T} / \mathrm{R}$ switch is needed, differential operation is required. By changing the buffer into an inverting Schmitt trigger and using the presented driver in conjunction, it is possible to operate differentially. It does, however, require twice the amount of components. Given that the drivers are placed outside the bore of the scanner this should not pose a problem. Additionally, since the driver is made using readily available components, the cost is in the range of $30-50 €$ per piece. Thus, the added cost of operating differentially is fairly low, even though the relative price doubles.

\section{Conclusion}

A PIN diode driver using readily available discrete components featuring forward and reverse bias switching times of $2 \mu \mathrm{s}$ and $0.4 \mu \mathrm{s}$, respectively, has been presented. When the driver is in forward bias, a current limited voltage is supplied to either a $\mathrm{T} / \mathrm{R}$ switch or Q-spoiling circuit. In reverse bias, $-5 \mathrm{~V}$ is supplied to the PIN diodes. The driver was tested in both an MRI scanner (for controlling a Q-spoiling switch on a receive surface coil) and NMR spectrometer (to control a T/R switch). The driver presented in this work had similar performance to the commercial solutions. Hence, a complete PIN diode driver has thus been presented which, to the best knowledge of the authors, have thus far been omitted in the MR community. Further, this paper takes a step towards 'build your own scanner/spectrometer'. Finally, utilizing a self-built driver eases the design of custom $\mathrm{T} / \mathrm{R}$ switches and Q-spoiling circuits since the built-in driver of the NMR spectrometer or MRI scanner is no longer a limiting factor. 


\section{Acknowledgments}

This work was supported by the Danish National Research Foundation [DNRF124].

\section{References}

[1] J. D. Sanchez-heredia, E. Søvsø, S. Hansen, C. Laustsen, V. Zhurbenko, J. H. Ardenkjær-larsen, Low-Noise Active Decoupling Circuit and its Application to 13C Cryogenic RF Coils at 3 T, Tomography 3 (1) (2017) 60-66.

[2] R. H. Caverly, PIN diode-based transmit-receive switch for 7 T MRI, in: IEEE Topical Conference on Biomedical Wireless Technologies, Networks, and Sensing Systems (BioWireleSS), 100-102, doi:10.1109/BIOWIRELESS.2016.7445574, 2016.

[3] R. H. Caverly, S. Benbrook, Control Device Thermal Modeling in MRI Receive Coil Q-Spoiling Circuits, IEEE Journal of Electromagnetics, RF and Microwaves in Medicine and Biology 1 (2) (2017) 52-58, ISSN 2469-7249, doi:10.1109/JERM. 2017.2776520 .

[4] R. H. Caverly, S. Benbrook, Thermal modeling of Q-spoil switching elements for MRI coils, in: IEEE MTT-S International Microwave Bio Conference (IMBIOC), 1-3, doi:10.1109/IMBIOC.2017.7965768, 2017.

[5] A. Maunder, M. Rao, F. Robb, J. M. Wild, Comparison of MEMS switches and PIN diodes for switched dual tuned RF coils, Magnetic Resonance in Medicine 80 (4) (2018) 1746-1753, doi:10.1002/mrm.27156.

[6] M. Twieg, M. A. de Rooij, M. A. Griswold, Active Detuning of MRI Receive Coils with GaN FETs, IEEE Trans. on Microw. Theory and Techn. 63 (12) (2015) 4169-4177, ISSN 0018-9480, doi:10.1109/TMTT.2015.2495366.

[7] B. Thapa, J. Kaggie, N. Sapkota, D. Frank, E.-K. Jeong, Design and Development of a General-Purpose Transmit/Receive (T/R) Switch for 3T MRI, Concepts in Magnetic Resonance Part B: Magnetic Resonance Engineering 46B (2) (2016) 56-65, doi:10.1002/cmr.b.21321.

[8] R. H. Caverly, X. Ma, Nonlinear stored charge vs d.c. bias-current relationship under high-level injection in pin diodes, Solid State Electron. 32 (4) (1989) 329-332, ISSN 00381101, doi:10.1016/0038-1101(89)90084-1.

[9] MA4P MELF \& HIPAX Series, MACOM Technology Solutions Inc., Rev. 16.

[10] Skyworks, Driver Circuit for High-Power PIN Diode Switches, Tech. Rep., Skyworks Solutions, Inc., 2016.

[11] National Semiconductor, P-I-N diode drivers, Tech. Rep., Electronic Engineering, 1972.

[12] National Semiconductor, PIN Diode Drivers, Tech. Rep., Application Note 49, 1986.

[13] J. Ardizzoni, Driving PIN Diodes: The Op-Amp Alternative, Analog Dialogue 44 (1) (2010) 11-15.

[14] G. Forlani, F. Fresia, Driving PIN Diodes: The Op-Amp Alternative, CSELT Rapporti tecnici 10 (5) (1982) 317-320.

[15] B. Harvey, A high-voltage ATE pin driver, in: Proc. of the Bipolar/BiCMOS Circuits and Tech. Meeting, 234-237, doi:10.1109/BIPOL.1992.274043, 1992.

[16] C. van Niekerk, P. W. van der Walt, High speed high voltage PIN diode driver, in: IEEE Intern. Symp. on Industrial Electronics, vol. 1, 167-170, doi:10.1109/ISIE.1998.707770, 1998.

[17] T. P. Ryan, W. Miller, Design and performance of a high speed driver circuit for PIN diode switches used in microwave hyperthermia, J. Biomed. Eng. 11 (2) (1989) 130-132.

[18] Y. Xiao, Z. Zhao, Z. Qian, H. Zhou, A High Isolation Switching Unit for MRI System, in: Procedia Eng., vol. 7, Elsevier Inc., ISSN 18777058, 265-269, doi:10.1016/j.proeng.2010.11.042, 2010.

[19] C. Brorsson, PIN diode drive circuits optimized for fast switching, Master's thesis, Chalmers, 2011.

[20] D. O. Brunner, L. Furrer, M. Weiger, W. Baumberger, T. Schmid, J. Reber, B. E. Dietrich, B. J. Wilm, R. Froidevaux, K. P. Pruessmann, Symmetrically biased T/R switches for NMR and MRI with microsecond dead time, J. Magn. Reson. 263 (2016) 147-155, ISSN 10960856, doi:10.1016/j.jmr.2015.12.016.

[21] D. H. Johansen, J. D. Sanchez-Heredia, J. R. Petersen, T. K. Johansen, V. Zhurbenko, J. H. Ardenkjær-Larsen, Cryogenic Preamplifiers for Magnetic Resonance Imaging, IEEE Trans. on Biomedical Circ. and Syst. 12 (1) (2018) 202-210, ISSN $1932-4545$.

[22] W. McMurray, Selection of Snubbers and Clamps to Optimize the Design of Transistor Switching Converters, IEEE Trans. on Industry Applications 16 (4) (1980) 513-523, ISSN 0093-9994, doi:10.1109/TIA.1980.4503823.

[23] R. J. Baker, B. P. Johnson, Stacking power MOSFETs for use in high speed instrumentation, Review of Scientific Instruments 63 (12) (1992) 5799-5801, doi:10.1063/1.1143366. 